\title{
Brain Oscillatory Coupling during Motor Control as a Potential Biomarker for Autism Spectrum Disorders: a comparative study
}

Kyung-min An ( $\square$ akmin@med.kanazawa-u.ac.jp )

Kanazawa University https://orcid.org/0000-0003-4093-8549

\section{Takashi lkeda}

Kanazawa University

\section{Tetsu Hirosawa}

Kanazawa University

Chiaki Hasegawa

Kanazawa University

\section{Yuko Yoshimura}

Kanazawa University

\section{Sanae Tanaka}

Kanazawa University

Daisuke N. Saito

Kanazawa University

Ken Yaoi

Kanazawa University

Sumie Iwasaki

Kanazawa University

Mitsuru Kikuchi

Kanazawa University

\section{Research}

Keywords: autism spectrum disorder, magnetoencephalography, motor cortex, brain oscillations, phaseamplitude coupling

Posted Date: July 8th, 2020

DOI: https://doi.org/10.21203/rs.3.rs-40571/v1

License: (c) (1) This work is licensed under a Creative Commons Attribution 4.0 International License.

Read Full License 


\section{Abstract}

Background Autism spectrum disorder (ASD) often involves dysfunction in general motor control and motor coordination, in addition to core symptoms. However, the neural mechanisms underlying motor dysfunction in ASD are poorly understood. To elucidate this issue, we focused on brain oscillations and their coupling in the primary motor cortex (M1). Methods We recorded magnetoencephalography in 18 children with autism spectrum disorder, aged 5 to 7 years, and 19 age- and IQ-matched typicallydeveloping children while they pressed button during a video-game-like motor task. We measured motorrelated gamma (70 to $90 \mathrm{~Hz}$ ) and pre-movement beta oscillations ( 15 to $25 \mathrm{~Hz}$ ) in the primary motor cortex. To determine the coupling between beta and gamma oscillations, we applied phase-amplitude coupling to calculate the statistical dependence between the amplitude of fast oscillations and the phase of slow oscillations. Results We observed a motor-related gamma increase and a pre-movement beta decrease in both groups. The autism spectrum disorder group exhibited a reduced motor-related gamma increase $(t(35)=2.412, p=0.021)$ and enhanced pre-movement beta decrease $(t(35)=2.705, p=0.010$ ) in the ipsilateral primary motor cortex. We found the phase-amplitude coupling that the high-gamma activity modulated by the beta rhythm in the primary motor cortex. Phase-amplitude coupling in the ipsilateral primary motor cortex was reduced in the autism spectrum disorder group compared with the control group $(t(35)=3.610, p=0.001)$. Using oscillatory changes and their coupling, linear discriminant analysis classified autism spectrum disorder and control groups with high accuracy (area under the receiver operating characteristic curve 97.1\%). Limitations Further studies with larger sample size and age range of data are warranted to confirm these effects. Conclusions The current findings revealed alterations in oscillations and oscillatory coupling reflecting the dysregulation of a motor gating mechanism in ASD. These results may be helpful for elucidating the neural mechanisms underlying motor dysfunction in ASD, suggesting the possibility of developing a biomarker for ASD diagnosis.

\section{Background}

Autism spectrum disorder (ASD) is a neurological developmental disorder characterized by persistent deficits in social interaction and communication, and the presence of repetitive behaviors with restricted interests (1). In addition to the core symptoms of ASD, previous studies have reported that children and adults with ASD often exhibit significant motor dysfunction (2-5). Kanner (1943), who proposed the first clear definition of autism, included motor dysfunction as a symptom of ASD (6). Motor abnormalities have been widely reported in ASD, involving fine and gross motor skills, gait, balance, and posture (2-4, 7-11). Although motor dysfunction is excluded from current diagnostic criteria for ASD, several recent studies have suggested that motor abnormalities should be considered a core symptom of $\operatorname{ASD}(2,3,12)$.

Brain oscillations are rhythmic patterns of neural activity, and motor control modulates typical brain oscillations, particularly in the beta $(13-30 \mathrm{~Hz})$ and gamma frequency bands $(60-90 \mathrm{~Hz})$. Gamma oscillations are known to increase immediately after movement onset, and these motor-related gamma oscillations are thought to be associated with motor execution and initiation (13-16). In addition, the power of beta oscillations begins to decrease prior to movement onset and is maintained during 
movement execution, and these pre-movement beta decreases are thought to be related to motor planning and movement preparation (16-19). In our previous study, we found that motor-related gamma oscillations were altered in children with ASD, suggesting a potential biomarker for ASD using gamma oscillatory changes as a neurophysiological index and button response time as a behavioral index (20). However, pre-movement beta oscillations are not well characterized in ASD. Thus, in the present study, we studied that motor dysfunction in ASD might be reflected by alterations in not only motor-related gamma oscillations but also pre-movement beta oscillations.

Recently, there has been increasing research interest in the coupling between brain oscillations, with several studies reporting that the particular phase of a low-frequency rhythm modulates the amplitude of high-frequency brain activity in several brain areas $(21-24)$. This phase-amplitude coupling (PAC) plays a functional role in local brain connectivity, coordinating the timing of brain activity in brain networks. In primary motor cortex (M1), the amplitude of high-gamma activity was reported to be modulated by the phase of the beta rhythms in previous electrocorticogram (ECoG) studies $(25,26)$. PAC has been suggested as a crucial gating mechanism for movement execution, and aberrant PAC has been reported in patients with movement disorders such as Parkinson's disease $(25,26)$. We speculate that beta-high gamma coupling from M1 might reflect the mechanisms underlying motor dysfunction in ASD. Importantly, previous studies have only investigated PAC from M1 using invasive brain recording techniques. Thus, we sought to investigate PAC from M1 using non-invasive neuroimaging methods.

In the present study, we measured beta-gamma PAC from M1 in children using child-customized magnetoencephalography (MEG). We hypothesized that beta-high gamma PAC from M1 would be altered in children with ASD. In addition, we investigated whether beta and gamma oscillations during motor control were altered in ASD. Moreover, by combining these physiological indices, we developed a potential biomarker for ASD.

\section{Methods}

\section{Participants}

Eighteen children with ASD (mean age $=6.00$ years, $S D=0.59$; five females) and 19 age-matched typically-developing (TD) children (mean age $=5.71$ years, $S D=0.46$, four females) participated in this study (Table 1). All participants were identified as right-handed using the Edinburgh Handedness Inventory (27). The Ethics Committee of the Kanazawa University Hospital approved this study, and parents of all participants provided full written informed consent. 
Table 1

Participants' characteristics.

\begin{tabular}{|lllll|}
\hline \multicolumn{1}{|c}{ TD } & ASD & $t$ & $p$ \\
\hline $\begin{array}{l}\text { Gender } \\
\text { (male/female) }\end{array}$ & $15 / 4$ & $13 / 5$ & & \\
\hline Age (months) & & & & \\
\hline K-ABC & $68.53 \pm 5.56$ & $72.00 \pm 7.10$ & -1.662 & 0.105 \\
achievement score & $104.21 \pm 13.34$ & $96.94 \pm 17.23$ & 1.439 & 0.159 \\
\hline $\begin{array}{l}\text { ADOS total score } \\
\text { Means } \pm \text { SDs and accompanying statistics (two-sided t-tests) of participants' characteristics. } \\
\text { Significant differences in age and intelligence were not observed between the TD and ASD groups. K- } \\
\text { ABC = Kaufman Assessment Battery for Children; ADOS = Autism Diagnostic Observation Schedule. }\end{array}$ \\
\hline
\end{tabular}

ASD participants had confirmed diagnoses of ASD based on DSM-V criteria for autism or Asperger syndrome (1), the Diagnostic Interview for Social and Communication Disorders (28), and/or the Autism Diagnostic Observational Schedule, Generic (ADOS) (4).

Intelligence was assessed using the Kaufman Assessment Battery for Children (K-ABC), and there was no significant difference in achievement scores between the two groups $(t(35)=1.439, p=0.159)$.

\section{Experimental paradigm}

To investigate motor-related oscillatory changes and oscillatory coupling, we used a video-game-like motor task developed in our previous study (20). The experimental paradigm for the video-game-like motor task is shown in Fig. 1A. The motor task contained 10 blocks, involving 100 button presses. The aim of this motor task is to help a puppy collect fruit by pressing a button. Participants were instructed to gaze at a fixation point in the middle of the screen. When the fruit appeared at the fixation point, participants were instructed to press the button using their right index finger. After the button press, the puppy jumped and caught the fruit. Fruit targets randomly appeared every 3.5 to $4.5 \mathrm{~s}$ after the button response. Each block contained 10 trials, and a bone with a red ribbon was obtained as a reward for successfully performing each block.

We designed this motor task to minimize participants' eye movement, by positioning the fixation point at the middle of the screen. To calculate response time, we instructed participants to press a button as quickly as possible. The button response was obtained using a non-magnetic fiber optic response pad (LUMINA LU400-PAIR, Cedrus Corporation, San Pedro, CA, USA).

MEG was recorded for approximately 9 min (100 button presses) during the motor task. The visual stimuli were presented on a screen $\left(26^{\circ} \times 21^{\circ}\right.$ of visual angle) using an LCD projector (IPSiO PJWX6170N, Ricoh Company, Ltd., Tokyo, Japan). 


\section{Magnetoencephalography recording}

MEG data were acquired using a whole-head 151-channel child-customized MEG system (PQ 1151 R, Yokogawa/KIT, Kanazawa, Japan) in a magnetically shielded room. MEG signals were digitized at $2000 \mathrm{~Hz}$ and low-pass filtered at $200 \mathrm{~Hz}$. MEG recording conditions were similar to those detailed in our previous studies (20). Prior to the experiment, we explained the experimental paradigm and procedures to participants and their parents. Participants practiced one block of the motor task to familiarize themselves with the experimental environment and paradigm. Participants were recorded in a comfortable supine position on a bed during MEG measurement, and two experimenters supported participants next to them to keep their attention to the task.

Four head positioning coils were attached to the scalp $(C z, 5 \mathrm{~cm}$ anterior from $\mathrm{Cz}$, and $5 \mathrm{~cm}$ from the superior side of the left and right pre-auricular points). The location of the positioning coils was measured before MEG recordings to calculate the position of the participant's head relative to the MEG sensors. The location of the positioning coils and information about head-shape were measured using a 3D digitizer (Fastrak, Polhemus, Colchester, VT, USA).

Brain structural images were individually obtained for source reconstruction from all participants using a 1.5 T magnetic resonance imaging (MRI) scanner (SIGNA Explorer, GE Healthcare, USA). For each participant, an MRI scan was acquired using the T1-weighted gradient echo and Silenz pulse sequence (repetition time $[T R]=435.68 \mathrm{~ms}$, echo time $[T E]=0.024 \mathrm{~ms}$, flip angle $=7^{\circ}$, field of view $[F O V]=220 \mathrm{~mm}$, matrix size $=256 \times 256$ pixels, slice thickness $=1.7 \mathrm{~mm}$, and 130 transaxial images).

\section{Data analysis}

MEG data were processed using the Brainstorm toolbox (29) and MATLAB (Mathworks, Natick, MA, USA). Data were band-pass filtered from 0.3 to $200 \mathrm{~Hz}$ and notch filtered at 60, 120, and $180 \mathrm{~Hz}$. We applied an independent component analysis method ("RunICA" implemented in Brainstorm, www.sccn.ucsd.edu/eeglab/) and removed the components representing heartbeats, eye blinks and eye movements identified by visual inspection based on time course and topography. After rejecting the components representing artifacts, the remaining components were back-projected into the signal space. We epoched data into segments from -3 to $3 \mathrm{~s}$ following button-press onset and selected successful trials.

We calculated weighted minimum norm estimates (wMNE) implemented in Brainstorm toolbox for source analysis (30-32). We estimated an overlapping sphere head model using individual MRI images and the noise-covariance matrix using the baseline period ( -2 to $-1.5 \mathrm{~s})$. We applied wMNE source localization using an overlapping conductor model with a Tikhonov regularization factor $(\lambda=0.1)$.

We calculated movement-related fields by averaging all trials according to the button-press onset and normalizing by the baseline period ( -2 to $-1.5 \mathrm{~s})$. We localized the cortical sources of the movementrelated fields using the wMNE method. We individually found the maximum peak of the bilateral cortical 
sources of the movement-related fields of each participant (Table 2) and used these cortical source data for further oscillatory analysis (Fig. 2A). 
Table 2

Individual button response time and bilateral source locations of motor-related fields.

\begin{tabular}{|c|c|c|c|c|c|c|c|}
\hline \multirow[t]{3}{*}{ Participant } & \multirow[t]{3}{*}{$\begin{array}{l}\text { Button response } \\
\text { time (ms) }\end{array}$} & \multirow{2}{*}{\multicolumn{3}{|c|}{$\begin{array}{l}\text { Left cortical source of motor- } \\
\text { related fields } \\
\text { (MNI coordinates) }\end{array}$}} & \multirow{2}{*}{\multicolumn{3}{|c|}{$\begin{array}{l}\text { Right cortical source of } \\
\text { motor-related fields } \\
\text { (MNI coordinates) }\end{array}$}} \\
\hline & & & & & & & \\
\hline & & $x$ & $Y$ & Z & $x$ & $\mathbf{Y}$ & Z \\
\hline
\end{tabular}

\section{TD children}

\begin{tabular}{|llllllll|}
\hline TD01 & 542.7 & -42.1 & 1.4 & 55.1 & 61.8 & 11.5 & 41.8 \\
\hline TD02 & 434.0 & -25.5 & -16.2 & 65.0 & 28.8 & -1.4 & 61.8 \\
\hline TD03 & 445.2 & -39.1 & -2.4 & 62.4 & 19.9 & -16.3 & 70.2 \\
\hline TD04 & 643.4 & -41.9 & 0.8 & 51.3 & 48.0 & 3.2 & 51.4 \\
\hline TD05 & 397.5 & -44.5 & -5.6 & 50.5 & 42.9 & -8.7 & 38.0 \\
\hline TD06 & 464.2 & -49.4 & -3.4 & 54.0 & 42.2 & 2.6 & 53.3 \\
\hline TD07 & 379.8 & -37.3 & -5.2 & 58.6 & 60.1 & -0.4 & 29.5 \\
\hline TD08 & 406.1 & -45.7 & -0.0 & 60.2 & 58.8 & -6.5 & 33.7 \\
\hline TD09 & 450.1 & -34.2 & -2.7 & 60.2 & 48.8 & -6.4 & 50.1 \\
\hline TD10 & 378.9 & -24.6 & -11.9 & 70.5 & 37.6 & -8.1 & 62.1 \\
\hline TD11 & 333.8 & -48.3 & 8.3 & 53.8 & 51.7 & 23.4 & 49.8 \\
\hline TD12 & 362.6 & -21.7 & -11.3 & 68.4 & 38.8 & -12.9 & 64.6 \\
\hline TD13 & 555.1 & -28.8 & -8.1 & 67.2 & 46.1 & -11.3 & 55.2 \\
\hline TD14 & 493.5 & -39.7 & -0.9 & 63.9 & 58.2 & -3.9 & 45.5 \\
\hline TD15 & 293.8 & -45.6 & 3.4 & 56.5 & 51.8 & -8.1 & 45.1 \\
\hline TD16 & 693.4 & -47.5 & 0.7 & 51.8 & 40.2 & 1.3 & 67.6 \\
\hline TD17 & 341.5 & -46.4 & -1.5 & 56.4 & 40.6 & -12.6 & 49.9 \\
\hline TD18 & 419.1 & -42.9 & -5.7 & 54.5 & 45.7 & 10.8 & 46.5 \\
\hline TD19 & 382.1 & -40.8 & 0.5 & 59.4 & 58.7 & -8.2 & 49.3 \\
\hline Mean & 442.99 & -39.3 & -3.1 & 58.9 & 46.4 & -2.7 & 50.8 \\
\hline SD & 104.16 & 8.5 & 5.8 & 5.9 & 11.0 & 9.9 & 11.1
\end{tabular}

Children with ASD 


\begin{tabular}{|c|c|c|c|c|c|c|c|}
\hline \multirow[t]{3}{*}{ Participant } & \multirow[t]{3}{*}{$\begin{array}{l}\text { Button response } \\
\text { time (ms) }\end{array}$} & \multirow{2}{*}{\multicolumn{3}{|c|}{$\begin{array}{l}\text { Left cortical source of motor- } \\
\text { related fields } \\
\text { (MNI coordinates) }\end{array}$}} & \multirow{2}{*}{\multicolumn{3}{|c|}{$\begin{array}{l}\text { Right cortical source of } \\
\text { motor-related fields } \\
\text { (MNI coordinates) }\end{array}$}} \\
\hline & & & & & & & \\
\hline & & $x$ & $Y$ & Z & $x$ & $Y$ & Z \\
\hline ASD01 & 519.6 & -47.1 & 9.7 & 49.2 & 43.0 & -11.8 & 56.5 \\
\hline ASD02 & 742.5 & -39.4 & -1.1 & 61.2 & 54.4 & -0.7 & 41.2 \\
\hline ASD03 & 714.0 & -37.5 & -2.3 & 59.4 & 52.6 & 7.2 & 42.7 \\
\hline ASD04 & 427.1 & -34.6 & -11.5 & 74.2 & 36.5 & -14.0 & 73.0 \\
\hline ASD05 & 495.8 & -37.4 & -16.2 & 61.3 & 45.2 & -2.5 & 52.0 \\
\hline ASD06 & 962.2 & -44.1 & -15.8 & 49.3 & 46.2 & -6.0 & 52.8 \\
\hline ASD07 & 540.4 & -46.6 & -5.3 & 2.7 & 36.4 & -31.4 & 15.7 \\
\hline ASD08 & 670.8 & -47.1 & 4.0 & 44.3 & 53.4 & -5.2 & 49.1 \\
\hline ASD09 & 724.5 & -39.3 & -11.0 & 52.6 & 30.7 & -3.7 & 56.5 \\
\hline ASD10 & 490.7 & -44.6 & -14.1 & 63.2 & 46.7 & 3.0 & 60.0 \\
\hline ASD11 & 398.1 & -55.1 & 0.1 & 44.7 & 36.4 & 5.9 & 48.4 \\
\hline ASD12 & 614.6 & -57.6 & -11.8 & 53.1 & 48.6 & 9.7 & 45.9 \\
\hline ASD13 & 599.3 & -27.0 & -6.0 & 69.0 & 39.9 & -13.4 & 49.6 \\
\hline ASD14 & 839.1 & -43.6 & -5.1 & 52.4 & 51.3 & -0.7 & 50.6 \\
\hline ASD15 & 639.6 & -51.9 & -4.5 & 39.3 & 36.7 & -12.3 & 60.6 \\
\hline ASD16 & 300.0 & -55.8 & -3.2 & 48.0 & 47.2 & -9.3 & 56.5 \\
\hline ASD17 & 524.9 & -47.4 & 2.5 & 52.3 & 61.2 & 7.8 & 39.7 \\
\hline ASD18 & 628.0 & -48.3 & 11.4 & 11.5 & 53.7 & 25.3 & 10.2 \\
\hline Mean & 601.73 & -44.3 & -5.9 & 51.5 & 44.1 & -5.3 & 50.7 \\
\hline$S D$ & 161.57 & 8.3 & 7.2 & 16.1 & 7.2 & 10.2 & 12.1 \\
\hline
\end{tabular}

To determine the motor-related oscillatory changes, each single trial source data were used to calculate the time-frequency representations (TFRs) using a 7-cycle Morlet wavelet. We converted TFRs to the percentage change in power relative to the baseline period. TFRs of each trial source were averaged within participants and group-averaged across TD and ASD participants, respectively. We observed that motor-related gamma oscillations increased from 70 to $90 \mathrm{~Hz}$ during 0 to $100 \mathrm{~ms}$ and pre-movement beta 
oscillations decreased from $15 \mathrm{~Hz}$ to $25 \mathrm{~Hz}$ during -200 to $0 \mathrm{~ms}$ according to the button response. We calculated the power changes of motor-related gamma oscillations and pre-movement beta oscillations by averaging power values in these time and frequency windows.

To estimate the phase-amplitude coupling between the beta and gamma oscillations, we used Mean Vector Length analysis implemented in the Brainstorm toolbox $(21,33)$. To calculate PAC, the data length containing 10 cycles of the lowest frequency is required. Thus, we selected a 1-s time window from $200 \mathrm{~ms}$, which is the start of the pre-movement beta decrease, until $800 \mathrm{~ms}$. This time window covered motor-related gamma increases and pre-movement beta decreases. We calculated PAC between the phase at 13 to $30 \mathrm{~Hz}$ and amplitude at 30 to $200 \mathrm{~Hz}$, in the time window during motor control ( -200 to $800 \mathrm{~ms}$ ). The statistical dependence that the gamma band activity is modulated by the beta rhythm was valued as the modulation index.

We used the power changes of the motor-related gamma oscillations and pre-movement beta oscillations, and beta-gamma coupling in the bilateral M1 for further statistical analysis.

\section{Statistical analysis}

We used SPSS version 24.0 (IBM Corporation, New York, USA) for statistical analysis. We applied twosample t-tests (two-tailed) to compare differences in the participant characteristics (age, K-ABC scores, button response time) between the TD and ASD groups. To test our hypothesis, we compared power changes in motor-related gamma and pre-movement beta oscillations, and beta-gamma coupling between two groups using two-sample t-tests (two-tailed). We employed an alpha level of 0.05 for all statistical analyses.

To investigate the ability that both behavioral and neural indices classify participants into the TD and ASD groups, we performed Fisher's linear discriminant analysis with leave-one-out cross-validation test.

To test the predictive accuracy of the classification method, we employed behavioral and brain oscillatory indices representing significant differences between groups. First, we tested the predictive accuracy of PAC using linear discriminant analysis with button response time and age. And we additionally added brain oscillatory indices (i.e. ipsilateral beta power and gamma power) into the discriminant analysis to test whether the predictive accuracy would be higher. For the cross-validation test, each case was derived from all other cases, and the remaining cases were classified. We analyzed the receiver operator characteristic (ROC) curves using sensitivity and 1 - specificity from the result of a linear discriminant analysis. Participants' discriminative capacity was determined by the area under the ROC curve (AUC).

\section{Results}

\section{Button response time}

We selected trials on which participants pressed the button within 200 to $2000 \mathrm{~ms}$ after the visual target onset, to exclude failed and accidental button responses. Individual button response time was considered 
as the latency between the visual trigger and button-press onset (Table 2).

The mean response time of the TD group was $443.0 \pm 104.2 \mathrm{~ms}$ (mean \pm SD), and the ASD group showed $601.7 \pm 161.6 \mathrm{~ms}$ (mean $\pm \mathrm{SD}$ ) of the mean response time. Consistent with our previous study, the ASD group exhibited a significantly longer mean response time than the TD group $(t(35)=-3.572, p=0.001)$ (Fig. 1B).

\section{Motor-related oscillatory changes}

Figure 2B shows the group averaged TRFs from the individual bilateral peak source of Motor fields in the 19 TD children and 18 children with ASD. Gamma oscillatory power increased in the 70 to $90 \mathrm{~Hz}$ range immediately after the button press onset. The decrease of beta oscillatory power in the 15 to $25 \mathrm{~Hz}$ range was started approximately $200 \mathrm{~ms}$ prior to the button press onset.

We observed diminished gamma power increase in the ASD group than for the TD group in the ipsilateral M1 $(t(35)=2.412, p=0.021)$, but not in the contralateral M1 $(p>0.05)$ (Fig. 2C). In contrast, the premovement beta power decrease was significantly enhanced in the ASD group compared with the TD group in the ipsilateral M1 $(t(35)=2.705, p=0.010)$, but not in the contralateral M1 $(p>0.05)$ (Fig. 2D).

\section{Motor-related phase-amplitude coupling}

We calculated the phase-amplitude coupling between beta (13 to $30 \mathrm{~Hz}$ ) and gamma (40 to $200 \mathrm{~Hz}$ ) frequency ranges. We found PAC signals between beta $(13 \mathrm{~Hz})$ and high-gamma oscillations (100 to $140 \mathrm{~Hz}$ ) in bilateral M1 in the TD and ASD groups (Fig. 3A).

The ASD group showed significantly lower PAC signals than the TD group for ipsilateral M1 $(t(35)=$ 3.610, $p=0.001$ ), but not contralateral M1 ( $p>0.05$ ) (Fig. 3B).

\section{Classification using linear discriminant analysis}

We found significant differences in the button response time, ipsilateral beta and gamma oscillations, and beta-gamma PAC between TD and ASD groups. To investigate the efficiency of the classifier for distinguishing between the two groups, we conducted a linear discriminant analysis using these variables. Using ipsilateral PAC with response time and age, we found that a linear discriminant analysis classifier identified participants in the two groups with $83.8 \%$ accuracy $(72.2 \%$ sensitivity and $94.7 \%$ specificity) (Fig. 4A). Adding pre-movement beta decrease with age correction enabled the identification of a more effective classifier, with $86.5 \%$ accuracy ( $88.9 \%$ sensitivity and $84.2 \%$ specificity). The ROC curve confirmed the predictive ability of the method, with an AUC of $97.1 \%$ (Fig. 4B).

\section{Discussion}

To the best of our knowledge, this is the first non-invasive study to investigate the coupling between distinct frequency oscillations in the M1. Using MEG, we examined oscillatory changes and oscillatory coupling in $\mathrm{M} 1$ to investigate the neural mechanisms underlying motor dysfunction in ASD. We confirmed 
a prolonged response time during the motor task in the ASD group compared with the TD group. In addition, we observed alterations in motor-related gamma oscillations and pre-movement beta oscillations in the ASD group. Oscillatory coupling, by which the beta rhythm modulated high-gamma activity, was reduced in the ASD group. In addition, using motor behavior and motor-related oscillatory changes and oscillatory coupling, we developed a potential biomarker for ASD.

\section{Button response time}

We confirmed that the ASD group exhibited a button response time that was prolonged by approximately 150 ms compared with the TD group. The finding of an increased response time is in accord with previous behavioral studies. A number of previous studies have reported that ASD individuals exhibited broad motor deficits in gross and fine motor skills, posture, gait, and balance $(2,3,5,7-11)$. The prolonged button response time of children with ASD might be related to their dysfunctions in the motor.

\section{Motor-related oscillatory changes}

We observed an increase in gamma oscillations and a decrease of beta oscillations in the bilateral M1 of TD and ASD groups. The results revealed that the ASD group exhibited alterations in the motor-related gamma increase and pre-movement beta decrease in M1.

We confirmed that motor-related gamma oscillations were reduced in ASD, consistent with our previous findings (20). The power of gamma oscillations is reported to be increased immediately after movement onset in adults $(13,15)$ and children $(34,35)$. This transient gamma increase is thought to be related to the initiation of movement and motor execution (13-15). In the present study, we ascertain the evidence that altered motor-related gamma activity reflects impaired movement initiation in ASD.

In addition, we found that the finger movement suppressed the power of pre-movement beta oscillations in both groups. Adult participants exhibited a pre-movement beta power decrease approximately 2 to $1 \mathrm{~s}$ prior to movement onset during self-paced movement $(16,17,19,36)$ and pre-cued motor tasks $(18,35$, 37). In the current study, children showed a pre-movement beta decrease starting at approximately $200 \mathrm{~ms}$ prior to movement onset. This late latency of pre-movement beta decreases is consistent with the results of previous MEG studies in children (34). We observed significantly enhanced pre-movement beta oscillations before movement onset in the ASD group. Pre-movement beta decreases are thought to be related to movement planning and movement preparation for appropriate motor responses $(18,36,37)$. The enhanced pre-movement beta changes in the ASD group might reflect a requirement for stronger brain activity compared with the TD group, to compensate for movement deficits.

\section{Motor-related phase-amplitude coupling}

In the current MEG study, the results revealed oscillatory coupling, by which high gamma brain activity at 100 to $140 \mathrm{~Hz}$ was modulated by the beta rhythm at $13 \mathrm{~Hz}$ in M1 in human children. The frequency range of the oscillatory coupling was different from the frequency band of the beta power changes (i.e. 15 to $25 \mathrm{~Hz}$ ) and the gamma power changes (i.e. 70 to $90 \mathrm{~Hz}$ ). The different frequency ranges between the 
oscillatory power changes and oscillatory coupling might reflect their different underlying mechanisms and indirect relationship and different underlying mechanisms.

This beta-high gamma coupling measured by MEG in the present study is consistent with PAC patterns revealed using invasive local field potential measurement techniques. Previous ECoG studies reported coupling between the amplitude of high-gamma activity and the phase of the beta rhythm in human M1 $(25,26)$. Cross-frequency coupling has also been observed between the gamma activity from the M1 and the low-frequency oscillations from the ventral intermediate nucleus (i.e. the motor nucleus of the thalamus) (38). Thalamo-cortical PAC has been suggested to play a role as a mechanism for gating motor behavior. Therefore, our finding that the slow rhythm modulates fast brain activity in M1 might reflect an underlying gating mechanism for motor execution, and could be related to distal communication mediated by the thalamus across the thalamo-cortical motor network.

It has been suggested that high-frequency brain activity, such as gamma oscillation, is related to local cortical processing over relatively short spatial scales $(24,39,40)$ and is correlated with blood-oxygenlevel-dependent signals (41-43). Given conduction delays, in contrast, low-frequency oscillations are thought to transfer information over large spatial scales by coordinating activities in distinct cortical areas $(21,22,24,44,45)$. Therefore, the coupling between slow- and fast-frequency oscillations has been proposed as a mechanism for local connectivity by coordinating activity in distributed brain regions across multiple spatial and temporal scales $(21,22,24)$.

In the present study, we found that beta-high gamma coupling was reduced in M1 in the ASD group. These findings are consistent with previous studies reporting that individuals with ASD exhibit reduced PAC during visual gating tasks $(46)$ and face recognition tasks $(47,48)$. Previous studies have proposed that aberrant PAC in ASD is linked to dysregulation of local connectivity and sensory processing. The current findings suggest that reduced PAC in the M1 might reflect atypical local brain connectivity and dysregulated motor processing in ASD.

We could find the atypical brain oscillations and oscillatory coupling in the ipsilateral hemisphere from the ASD group. The previous functional MRI studies have revealed that the ipsilateral motor cortex activity was related to the the execution of complex movements (49-51). The ipsilateral motor cortex has been also reported to play a role in motor learning (52) and controlling the timing of hand muscle recruitment (53). From these evidences, the altered brain oscillations and oscillatory coupling in the ipsilateral hemisphere of the ASD group may indicate their dysfunctions in the coordination of the precise and complex movements.

In addition, we investigated potential biomarkers for ASD using behavioral and neurophysiological oscillatory indices, revealing that linear discriminant analysis was able to classify these two groups. Although ASD involves various symptoms, we found that brain oscillations and oscillatory coupling during motor control discriminated between ASD participants and TD participants with high accuracy. While ASD is assumed to have various pathophysiology, the current finding that motor-related indices discriminated with high accuracy revealed that motor dysfunction is a common and important 
component of ASD. These findings support the notion that motor dysfunction in ASD should be considered an important characteristic of ASD.

\section{Limitations}

These findings were obtained from a relatively small number of participants. To reduce age-related bias, we recruited participants within a narrow age range (4 to 7 years). Future studies should examine a larger sample size and age range of participants to improve the reliability of the current findings.

\section{Conclusions}

To the best of our knowledge, this is the first non-invasive study to investigate cross-frequency coupling in M1. Coupling between distinct oscillations in M1 has previously been observed only using local field potentials measured with invasive ECoG recording. In the present study, we demonstrated that the beta rhythm modulated high-gamma activity in M1 using non-invasive MEG recording. In addition, the current results revealed that beta-high gamma coupling was reduced in the ASD group. This finding extends current understanding of motor gating dysfunction in ASD. In addition, we confirmed aberrant motorrelated gamma activity and found enhanced pre-movement beta power during motor control in ASD. These findings provide neurophysiological evidence for the dysfunction of motor initiation and motor preparation in ASD. In addition, these findings could be applied in the future studies about interventions or neurofeedback training for ASD.

\section{Abbreviations}

ADOS

Autism Diagnostic Observational Schedule; ASD:Autism Spectrum Disorder; K-ABC:Kaufman Assessment Battery for Children; M1:Primary motor cortex; MEG:Magnetoencephalography; PAC:Phase-amplitude coupling; TD:Typically developing

\section{Declarations}

\section{Ethics approval and consent to participate}

This study was approved by the Ethics Committee of the Kanazawa University Hospital. After a complete explanation of the study, parents of all participants provided full written informed consent.

\section{Consent for publication}

Not applicable.

\section{Availability of data and materials}


The datasets generated and/or analysed during the current study are not publicly available as they contain information that could compromise the privacy of research participants but are available from the corresponding author on reasonable request.

\section{Competing interests}

The authors declare that they have no competing interests.

\section{Funding}

This work was supported by a grant from the Center of Innovation Program from the Japan Science and Technology Agency (https://www.coistream.osaka-u.ac.jp/en) and KAKENHI Grant-in-Aid for Early-Career Scientists from the Japan Society for the Promotion of Science (grant number 20K16622). The funder had no role in the study design, data collection and analysis, decision to publish, or preparation of the manuscript.

\section{Authors' contributions}

KA designed and conceptualised the study, analysed the data, drafted the manuscript and revised the manuscript. TI, YY, DNS, KY, and SI recorded neuroimaging data and revised the manuscript. $\mathrm{CH}$ and ST derived autism spectrum disorders ascertainment and recorded neuroimaging and behavioural data. TH derived autism spectrum disorders ascertainment and revised the manuscript. MK secured funding, designed and conceptualised the study, derived autism spectrum disorders ascertainment, drafted the manuscript and revised the manuscript.

\section{Acknowledgements}

The authors thank Sachiko Kitagawa and Yukiko Saotome for conducting the behavioural and MEG experiments. They also thank Mutsumi Ozawa and Yoko Morita for preparing the experiments. The children and their parents who participated in this study are particularly appreciated.

\section{References}

1. Association AP. Diagnostic and statistical manual of mental disorders (DSM-5®): American Psychiatric Pub; 2013.

2. Fournier KA, Hass CJ, Naik SK, Lodha N, Cauraugh JH. Motor coordination in autism spectrum disorders: a synthesis and meta-analysis. J Autism Dev Disord. 2010;40(10):1227-40. 
3. Lloyd M, MacDonald M, Lord C. Motor skills of toddlers with autism spectrum disorders. Autism. 2013;17(2):133-46.

4. Lord C, Risi S, Lambrecht L, Cook EH Jr, Leventhal BL, DiLavore PC, et al. The autism diagnostic observation schedule-generic: a standard measure of social and communication deficits associated with the spectrum of autism. J Autism Dev Disord. 2000;30(3):205-23.

5. Teitelbaum P, Teitelbaum O, Nye J, Fryman J, Maurer RG. Movement analysis in infancy may be useful for early diagnosis of autism. Proc Natl Acad Sci U S A. 1998;95(23):13982-7.

6. Kanner L. Autistic disturbances of affective contact. Nervous child. 1943;2(3):217-50.

7. Bryson SE, Zwaigenbaum L, Brian J, Roberts W, Szatmari P, Rombough V, et al. A prospective case series of high-risk infants who developed autism. J Autism Dev Disord. 2007;37(1):12-24.

8. Jansiewicz EM, Goldberg MC, Newschaffer CJ, Denckla MB, Landa R, Mostofsky SH. Motor signs distinguish children with high functioning autism and Asperger's syndrome from controls. J Autism Dev Disord. 2006;36(5):613-21.

9. Molloy CA, Dietrich KN, Bhattacharya A. Postural stability in children with autism spectrum disorder. J Autism Dev Disord. 2003;33(6):643-52.

10. Noterdaeme M, Mildenberger K, Minow F, Amorosa $\mathrm{H}$. Evaluation of neuromotor deficits in children with autism and children with a specific speech and language disorder. Eur Child Adolesc Psychiatry. 2002;11(5):219-25.

11. Radonovich KJ, Fournier KA, Hass CJ. Relationship between postural control and restricted, repetitive behaviors in autism spectrum disorders. Front Integr Neurosci. 2013;7:28.

12. London EB. Categorical diagnosis: a fatal flaw for autism research? Trends Neurosci. 2014;37(12):683-6.

13. Cheyne D, Bells S, Ferrari P, Gaetz W, Bostan AC. Self-paced movements induce high-frequency gamma oscillations in primary motor cortex. Neuroimage. 2008;42(1):332-42.

14. Cheyne D, Ferrari P. MEG studies of motor cortex gamma oscillations: evidence for a gamma "fingerprint" in the brain? Front Hum Neurosci. 2013;7:575.

15. Muthukumaraswamy SD. Functional properties of human primary motor cortex gamma oscillations. J Neurophysiol. 2010;104(5):2873-85.

16. Pfurtscheller G, Graimann B, Huggins JE, Levine SP, Schuh LA. Spatiotemporal patterns of beta desynchronization and gamma synchronization in corticographic data during self-paced movement. Clin Neurophysiol. 2003;114(7):1226-36.

17. Bai O, Rathi V, Lin P, Huang D, Battapady H, Fei DY, et al. Prediction of human voluntary movement before it occurs. Clin Neurophysiol. 2011;122(2):364-72.

18. Doyle LM, Yarrow K, Brown P. Lateralization of event-related beta desynchronization in the EEG during pre-cued reaction time tasks. Clin Neurophysiol. 2005;116(8):1879-88.

19. Pfurtscheller G, Lopes da Silva FH. Event-related EEG/MEG synchronization and desynchronization: basic principles. Clin Neurophysiol. 1999;110(11):1842-57. 
20. An KM, Ikeda T, Yoshimura Y, Hasegawa C, Saito DN, Kumazaki H, et al. Altered Gamma Oscillations during Motor Control in Children with Autism Spectrum Disorder. J Neurosci. 2018;38(36):7878-86.

21. Canolty RT, Edwards E, Dalal SS, Soltani M, Nagarajan SS, Kirsch HE, et al. High gamma power is phase-locked to theta oscillations in human neocortex. Science. 2006;313(5793):1626-8.

22. Canolty RT, Knight RT. The functional role of cross-frequency coupling. Trends Cogn Sci. 2010;14(11):506-15.

23. Hyafil A, Giraud AL, Fontolan L, Gutkin B. Neural Cross-Frequency Coupling: Connecting Architectures, Mechanisms, and Functions. Trends Neurosci. 2015;38(11):725-40.

24. Jensen O, Colgin LL. Cross-frequency coupling between neuronal oscillations. Trends Cogn Sci. 2007;11(7):267-9.

25. de Hemptinne C, Ryapolova-Webb ES, Air EL, Garcia PA, Miller KJ, Ojemann JG, et al. Exaggerated phase-amplitude coupling in the primary motor cortex in Parkinson disease. Proc Natl Acad Sci U S A. $2013 ; 110(12): 4780-5$.

26. de Hemptinne C, Swann NC, Ostrem JL, Ryapolova-Webb ES, San Luciano M, Galifianakis NB, et al. Therapeutic deep brain stimulation reduces cortical phase-amplitude coupling in Parkinson's disease. Nat Neurosci. 2015;18(5):779-86.

27. Oldfield RC. The assessment and analysis of handedness: the Edinburgh inventory. Neuropsychologia. 1971;9(1):97-113.

28. Wing L, Leekam SR, Libby SJ, Gould J, Larcombe M. The Diagnostic Interview for Social and Communication Disorders: background, inter-rater reliability and clinical use. J Child Psychol Psychiatry. 2002;43(3):307-25.

29. Tadel F, Baillet S, Mosher JC, Pantazis D, Leahy RM. Brainstorm: a user-friendly application for MEG/EEG analysis. Comput Intell Neurosci. 2011;2011:879716.

30. Hamalainen MS, IImoniemi RJ. Interpreting magnetic fields of the brain: minimum norm estimates. Med Biol Eng Comput. 1994;32(1):35-42.

31. Hauk O. Keep it simple: a case for using classical minimum norm estimation in the analysis of EEG and MEG data. Neuroimage. 2004;21(4):1612-21.

32. Lin FH, Witzel T, Ahlfors SP, Stufflebeam SM, Belliveau JW, Hamalainen MS. Assessing and improving the spatial accuracy in MEG source localization by depth-weighted minimum-norm estimates. Neuroimage. 2006;31(1):160-71.

33. Tort AB, Komorowski R, Eichenbaum H, Kopell N. Measuring phase-amplitude coupling between neuronal oscillations of different frequencies. J Neurophysiol. 2010;104(2):1195-210.

34. Cheyne D, Jobst C, Tesan G, Crain S, Johnson B. Movement-related neuromagnetic fields in preschool age children. Hum Brain Mapp. 2014;35(9):4858-75.

35. Gaetz W, Macdonald M, Cheyne D, Snead OC. Neuromagnetic imaging of movement-related cortical oscillations in children and adults: age predicts post-movement beta rebound. Neuroimage. 2010;51(2):792-807. 
36. Stancak A Jr, Pfurtscheller G. Event-related desynchronisation of central beta-rhythms during brisk and slow self-paced finger movements of dominant and nondominant hand. Brain Res Cogn Brain Res. 1996;4(3):171-83.

37. Kaiser J, Birbaumer N, Lutzenberger W. Event-related beta desynchronization indicates timing of response selection in a delayed-response paradigm in humans. Neurosci Lett. 2001;312(3):149-52.

38. Opri E, Cernera S, Okun MS, Foote KD, Gunduz A. The Functional Role of Thalamocortical Coupling in the Human Motor Network. J Neurosci. 2019;39(41):8124-34.

39. Miller KJ, Leuthardt EC, Schalk G, Rao RP, Anderson NR, Moran DW, et al. Spectral changes in cortical surface potentials during motor movement. J Neurosci. 2007;27(9):2424-32.

40. Miller KJ, Zanos S, Fetz EE, den Nijs M, Ojemann JG. Decoupling the cortical power spectrum reveals real-time representation of individual finger movements in humans. J Neurosci. 2009;29(10):3132-7.

41. Mukamel R, Gelbard H, Arieli A, Hasson U, Fried I, Malach R. Coupling between neuronal firing, field potentials, and FMRI in human auditory cortex. Science. 2005;309(5736):951-4.

42. Niessing J, Ebisch B, Schmidt KE, Niessing M, Singer W, Galuske RA. Hemodynamic signals correlate tightly with synchronized gamma oscillations. Science. 2005;309(5736):948-51.

43. Scheeringa R, Fries P, Petersson KM, Oostenveld R, Grothe I, Norris DG, et al. Neuronal dynamics underlying high- and low-frequency EEG oscillations contribute independently to the human BOLD signal. Neuron. 2011;69(3):572-83.

44. Buzsaki G, Draguhn A. Neuronal oscillations in cortical networks. Science. 2004;304(5679):1926-9.

45. von Stein A, Sarnthein J. Different frequencies for different scales of cortical integration: from local gamma to long range alpha/theta synchronization. Int J Psychophysiol. 2000;38(3):301-13.

46. Seymour RA, Rippon G, Gooding-Williams G, Schoffelen JM, Kessler K. Dysregulated oscillatory connectivity in the visual system in autism spectrum disorder. Brain. 2019;142(10):3294-305.

47. Khan S, Gramfort A, Shetty NR, Kitzbichler MG, Ganesan S, Moran JM, et al. Local and long-range functional connectivity is reduced in concert in autism spectrum disorders. Proc Natl Acad Sci U S A. 2013;110(8):3107-12.

48. Mamashli F, Khan S, Bharadwaj H, Losh A, Pawlyszyn SM, Hamalainen MS, et al. Maturational trajectories of local and long-range functional connectivity in autism during face processing. Hum Brain Mapp. 2018;39(10):4094-104.

49. Buetefisch CM, Revill KP, Shuster L, Hines B, Parsons M. Motor demand-dependent activation of ipsilateral motor cortex. J Neurophysiol. 2014;112(4):999-1009.

50. Hummel F, Kirsammer R, Gerloff C. Ipsilateral cortical activation during finger sequences of increasing complexity: representation of movement difficulty or memory load? Clin Neurophysiol. 2003;114(4):605-13.

51. Seidler RD, Noll DC, Thiers G. Feedforward and feedback processes in motor control. Neuroimage. 2004;22(4):1775-83. 
52. Waters S, Wiestler T, Diedrichsen J. Cooperation Not Competition: Bihemispheric tDCS and fMRI Show Role for Ipsilateral Hemisphere in Motor Learning. J Neurosci. 2017;37(31):7500-12.

53. Davare M, Duque J, Vandermeeren $\mathrm{Y}$, Thonnard JL, Olivier E. Role of the ipsilateral primary motor cortex in controlling the timing of hand muscle recruitment. Cereb Cortex. 2007;17(2):353-62.

\section{Figures}
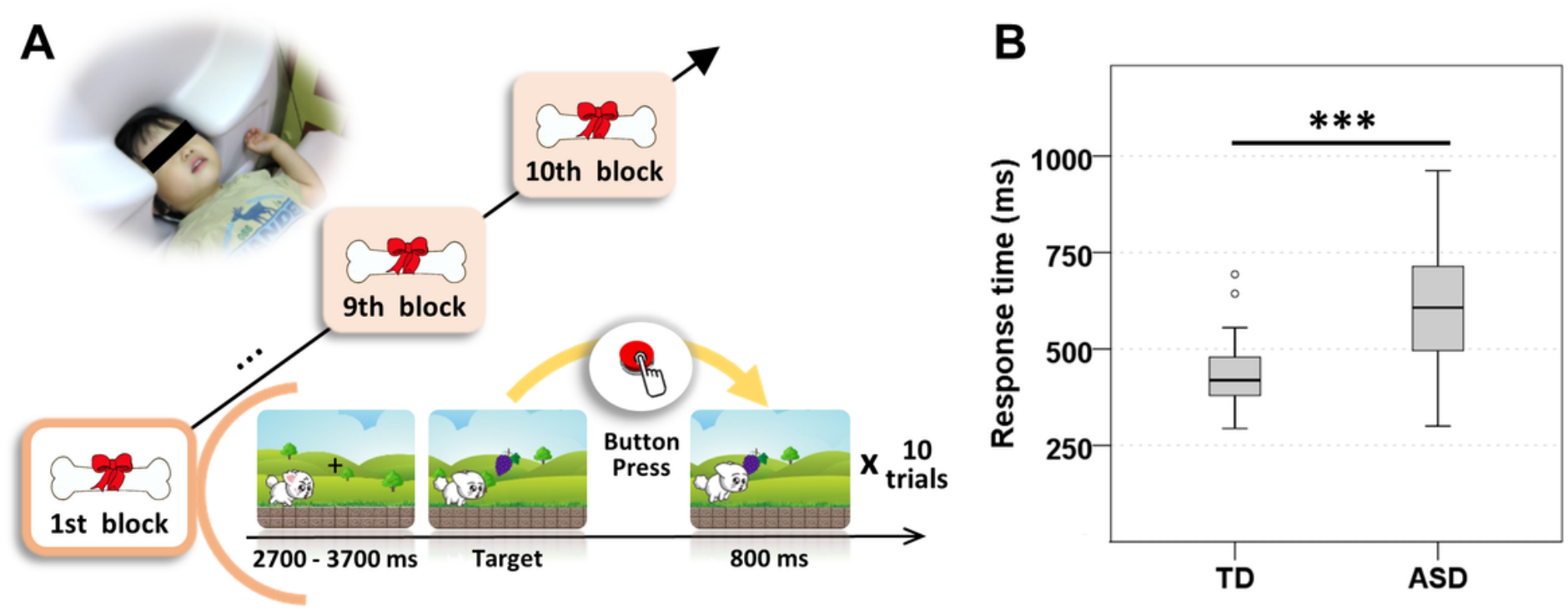

\section{Figure 1}

Experimental paradigm and button response time in the TD and ASD groups. a Children performed a video-game-like motor task. The motor task consisted of 10 blocks, and a bone with a red ribbon was given as a reward in the game after each block was successfully completed. The participants helped a puppy obtain fruit targets, and the action was repeated 10 times in each block. The target appeared every 3500 to $4500 \mathrm{~ms}$. Before the target appeared, participants were asked to pay attention to the fixation point. After the target appeared at the fixation point, participants were asked to press the button as quickly as possible. When the participant pressed the button, the puppy jumped and obtained a fruit target. $\mathrm{b}$ The button response time was calculated by subtracting the timing of the target from the timing of the button press. The ASD group showed a significantly prolonged button response time compared with the TD group $(t(35)=-3.572, p=0.001)$. 
A
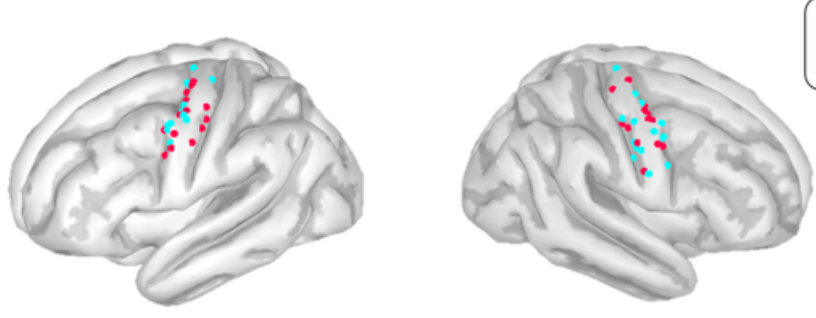

B

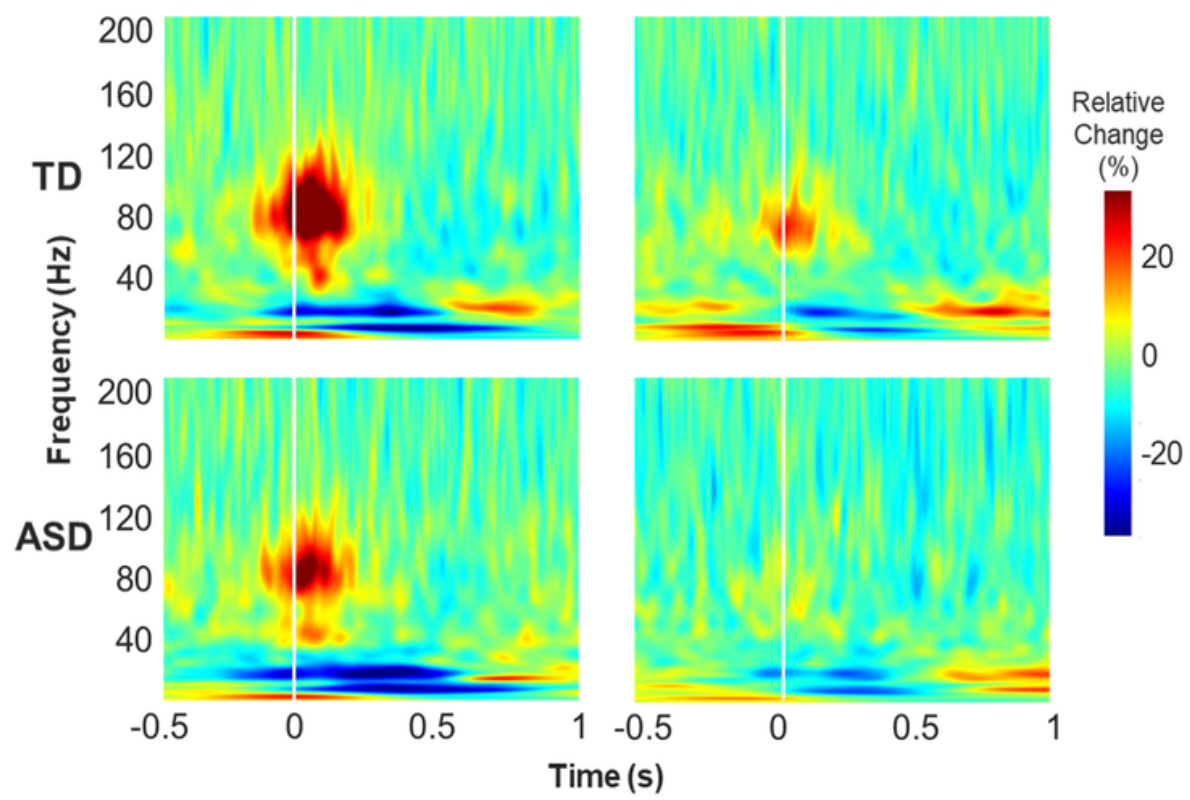

C < Motor-related Gamma >

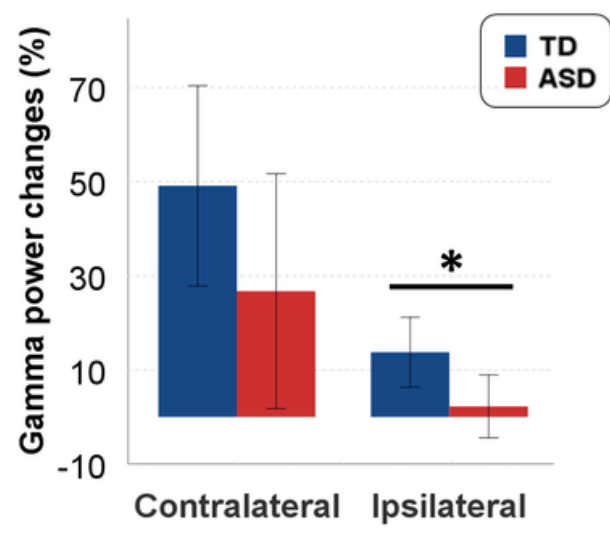

D < Pre-movement Beta >

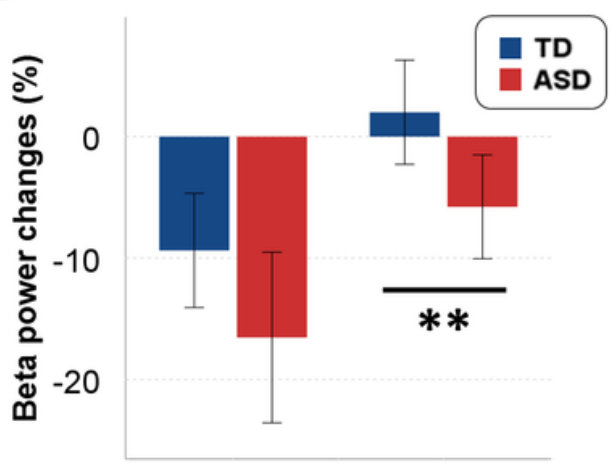

Contralateral Ipsilateral

Figure 2

Motor-related oscillatory changes in the TD and ASD groups. a Individual peak source of the motorrelated brain activity. Red dots indicate the source for children with ASD and blue dots indicate the source for TD children. b Time-frequency representations during motor control in the bilateral primary motor cortex (M1) in the TD and ASD groups. Motor-related gamma oscillations (70 to $90 \mathrm{~Hz}$ ) increased immediately after the button press. The pre-movement beta oscillations ( 15 to $25 \mathrm{~Hz}$ ) decreased $200 \mathrm{~ms}$ prior to the button press. c The ASD group showed a smaller gamma power increase than the TD group in the ipsilateral M1 $(t(35)=2.412, p=0.021)$, but not in the contralateral M1 $(p>0.05)$. $d$ The ASD group showed enhanced pre-movement beta decreases in ipsilateral M1 $(\mathrm{t}(35)=2.705, \mathrm{p}=0.010)$, but not in contralateral M1 ( $p>0.05)$. 
A

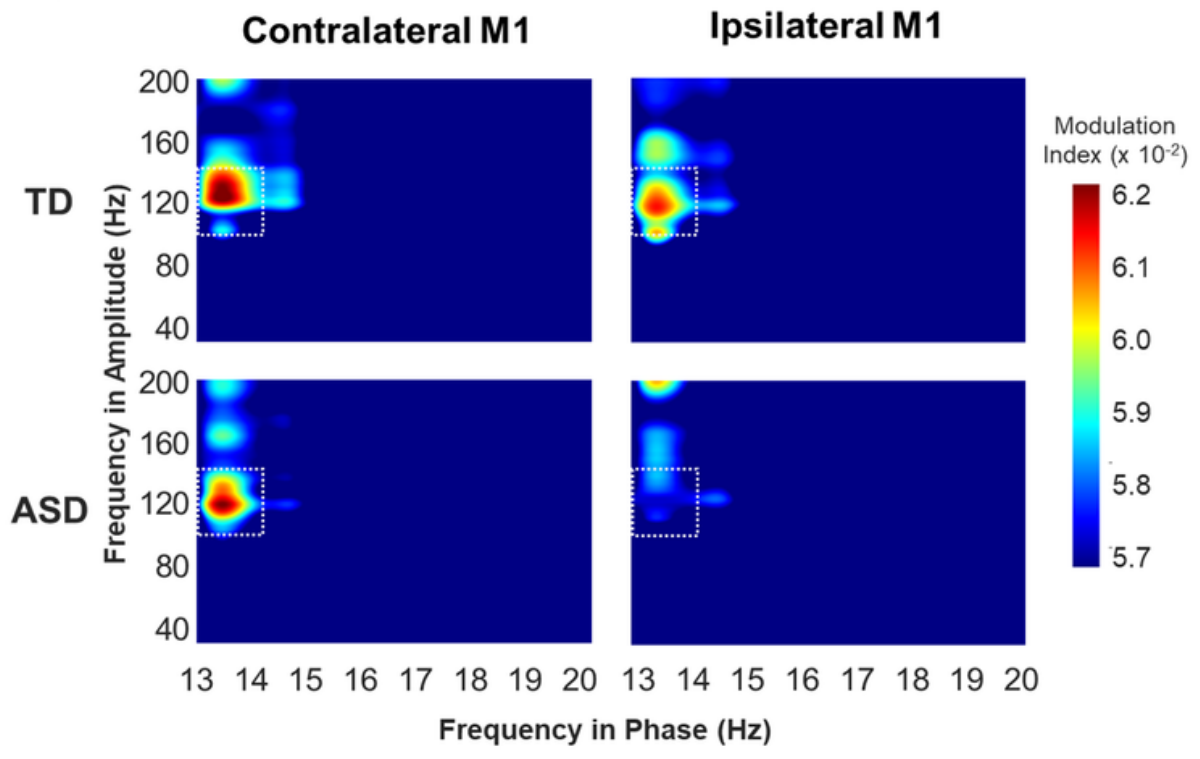

B

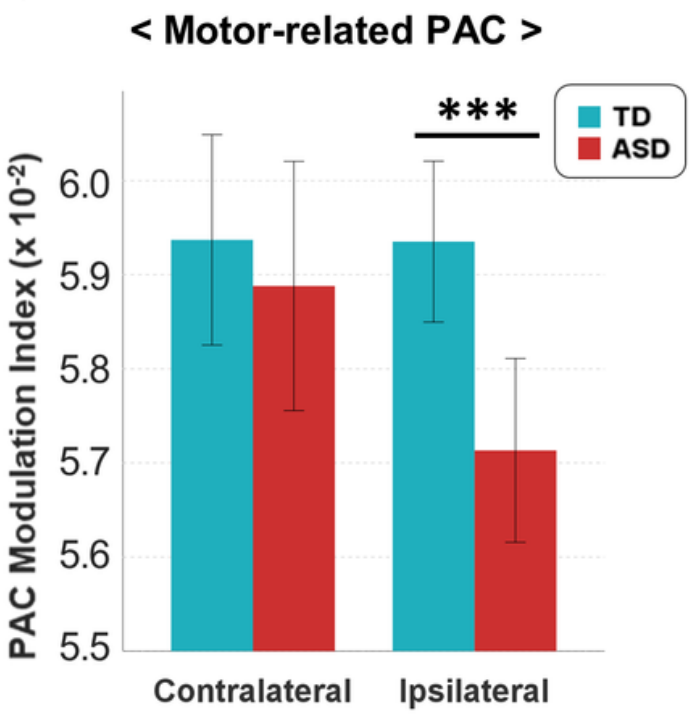

\section{Figure 3}

Phase amplitude coupling (PAC) in the bilateral primary motor cortex (M1). a During motor control, PAC between the low beta (approximately $13 \mathrm{~Hz}$ ) and high-gamma oscillations (100 to $140 \mathrm{~Hz}$ ) was observed in bilateral M1. b The modulation index of PAC in the contralateral M1 was no different between the two groups $(p>0.05)$. The ASD group exhibited significantly reduced beta-gamma PAC in ipsilateral M1 (t(35) $=3.610, p=0.001)$. 
A Linear Discriminant Analysis

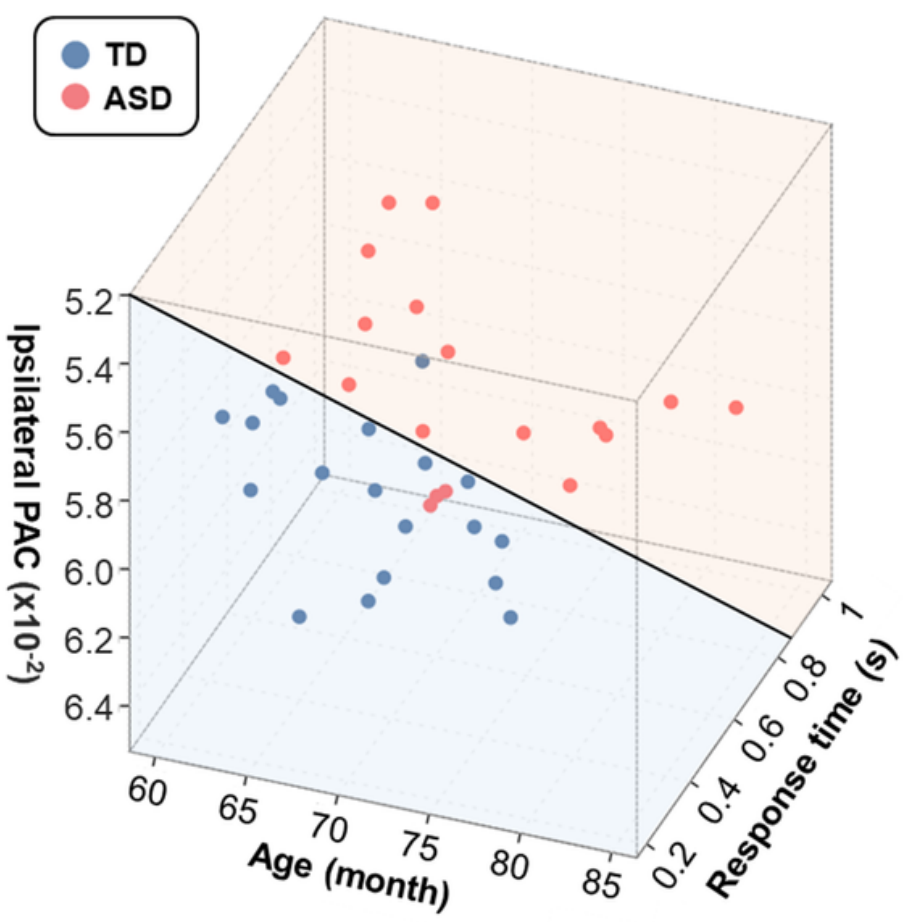

B $\quad$ ROC Curve $>$

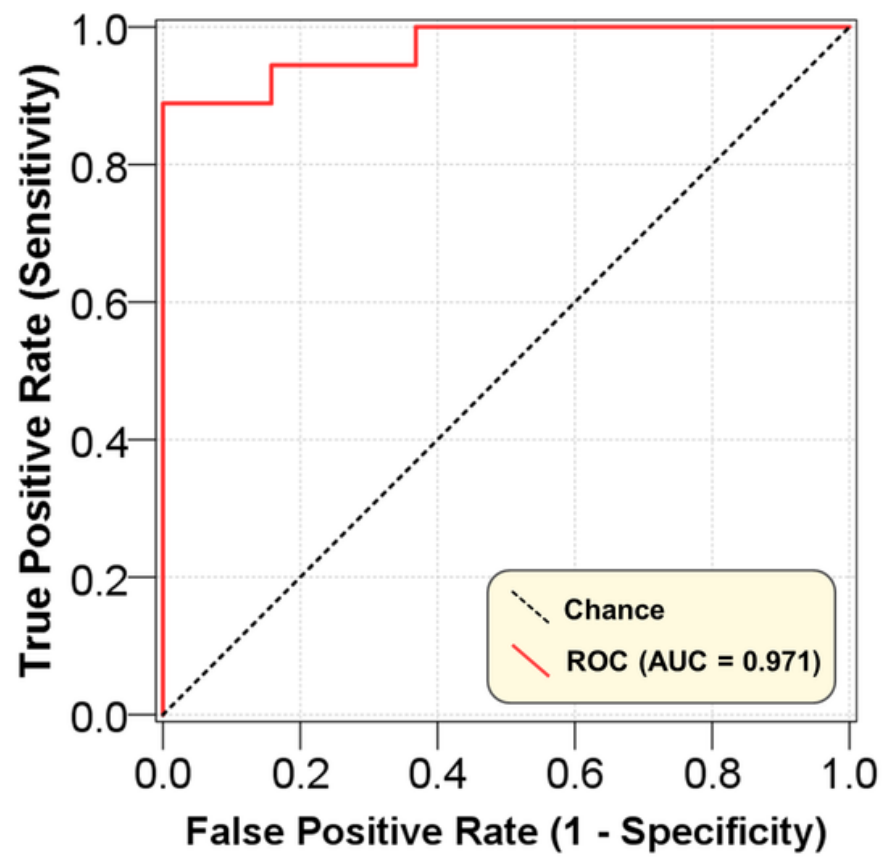

Figure 4

Linear discriminant analysis and receiver operating characteristic (ROC) curve using oscillations and oscillatory coupling parameters. a The linear discriminant analysis used ipsilateral PAC with response time and age. The discriminant classifier results exhibited accuracy of $83.8 \%$ (sensitivity $=72.2 \%$, specificity $=94.7 \%$ ) for blindly separating two groups. b Using ipsilateral PAC, ipsilateral beta and gamma power, response time and age, the ROC curve exhibited good discriminative capacity for two groups with an area under the ROC curve (AUC) value of 0.971 . 